\title{
New electrocardiographic criteria for the differentiation between counterclockwise and clockwise atrial flutter: correlation with electrophysiological study and radiofrequency catheter ablation
}

L P Lai, J L Lin, L J Lin, W J Chen, Y L Ho, Y Z Tseng, C H Chen, Y T Lee, W P Lien, S K S Huang

\begin{abstract}
Objective-To develop new electrocardiographic (ECG) criteria for the differentiation between counterclockwise and clockwise atrial flutters.

Background-Traditionally, the ECG differentiation between counterclockwise and clockwise atrial flutters is based on the flutter wave polarity in the inferior leads. However, determination of flutter wave polarity is subjective and sometimes difficult, especially in flutter waves of undulating pattern.
\end{abstract}

Patients-The study comprised 37 consecutive patients with drug resistant atrial flutter; 30 had counterclockwise and 17 had clockwise atrial flutter (10 had both forms of atrial flutter). The isthmus dependence was confirmed by entrainment study and catheter ablation. The ECG patterns of both types of atrial flutter were compared and the flutter wave polarity in the inferior leads was determined by four independent cardiologists. Results-The flutter wave polarity in the inferior leads appeared negative in 24, positive in one, and equivocal in five of the counterclockwise atrial flutters; polarity appeared negative in one, positive in 10, and equivocal in six of the clockwise atrial flutters. However, the aVF/lead I flutter wave amplitude ratio was $>2.5$ in all counterclockwise but $<2.5$ in all clockwise atrial flutters. The flutter wave nadirs in the inferior leads corresponded to the upstrokes in V1 in all counterclockwise atrial flutters, but corresponded to the downstrokes in V1 in all clockwise atrial flutters.

Conclusions-The flutter wave polarity in the inferior leads does not correlate well with the flutter wave rotating direction. However, counterclockwise and clockwise atrial flutters can be differentiated by new ECG criteria with high accuracy. (Heart 1998;80:80-85)

Keywords: atrial flutter; radiofrequency catheter ablation; electrocardiography

Atrial flutter was first described by Jolly and Ritchie in $1911 .^{1}$ This rhythm is characterised by flutter waves that appear as atrial complexes of constant morphology, polarity, and cycle length. Understanding of the mechanism of atrial flutter has grown rapidly in recent years. ${ }^{2-7}$ It is now well established that atrial flutter is a re-entrant rhythm and that the isthmus area between the inferior vena cava and the tricuspid annulus in the lower right atrium is a protected critical zone in most atrial flutters. These isthmus dependent atrial flutters can have either a counterclockwise or clockwise rotating circuit in the right atrium.

Atrial flutter with negative flutter waves in the inferior leads has been reported to have a counterclockwise rotating circuit in the right atrium. On the other hand, clockwise atrial flutter, which is also called the "mirror image" atrial flutter, might have positive flutter waves in the inferior leads because of a reversed activation sequence in the right atrium. However, electrocardiographic distinction between counterclockwise and clockwise atrial flutters based on such an observation might be difficult, particularly in the setting of rapid, undulating flutter waves.

Our study comprised 47 atrial flutter patterns observed in 37 consecutive patients during electrophysiological studies. The isthmus dependence and the flutter wave rotating direction were confirmed and the ECG patterns of counterclockwise and clockwise atrial flutters were compared. We propose new ECG criteria for the differentiation between counterclockwise and clockwise atrial flutters.

\section{Methods}

From September 1995 to August 1996, 37 consecutive patients with symptomatic, drug resistant atrial flutter underwent electrophysiological study and radiofrequency catheter ablation. There were 29 men and 8 women, with a mean (SD) age of 56 (15) years (range, 20-83). Table 1 shows the clinical characteristics of the patients.

\section{ELECTROPHYSIOLOGICAL STUDY}

Informed written consent was obtained from all patients. Patients underwent electrophysiological studies after antiarrhythmic medications had been discontinued for at least five half lives. Quadripolar electrode catheters were positioned at the high right atrium, the His bundle region, the right ventricular apex, and the 
Table 1 Characteristics of the patients and their atrial flutter

\begin{tabular}{|c|c|c|c|c|c|c|c|c|c|}
\hline \multirow[b]{2}{*}{ Patient } & \multirow{2}{*}{$\begin{array}{l}\text { Age } \\
\text { (years) }\end{array}$} & \multirow[b]{2}{*}{ Sex } & \multirow{2}{*}{$\begin{array}{l}\text { Associated heart disease or } \\
\text { arrhythmia }\end{array}$} & \multicolumn{2}{|l|}{$A F L C L$} & \multicolumn{2}{|c|}{ aVF/IFW ratio } & \multicolumn{2}{|c|}{$F W$ polarity } \\
\hline & & & & $C C W$ & $C W$ & $C C W$ & $C W$ & $C C W$ & $C W$ \\
\hline 1 & 58 & M & $\mathrm{HCM}$ & 206 & 196 & 4.8 & 1.8 & $\mathrm{n}$ & $\mathrm{p}$ \\
\hline 2 & 46 & $M$ & Atrial fibrillation & 188 & - & 5.0 & - & $\mathrm{n}$ & - \\
\hline 3 & 61 & $\mathrm{M}$ & & 288 & - & 2.6 & - & $\mathrm{n}$ & - \\
\hline 4 & 55 & M & Atrial fibrillation & 198 & - & 10.7 & - & $\mathrm{n}$ & - \\
\hline 5 & 71 & M & Atrial fibrillation & 188 & - & 2.8 & - & $\mathrm{p}$ & - \\
\hline 6 & 71 & M & & 243 & - & 9.3 & - & $\mathrm{n}$ & - \\
\hline 7 & 65 & M & AVRT & - & 200 & - & 1.2 & - & $\mathrm{e}$ \\
\hline 8 & 64 & $\mathrm{~F}$ & & 196 & - & 11.5 & - & $\mathrm{n}$ & - \\
\hline 9 & 45 & M & & 219 & - & 4.7 & - & $\mathrm{n}$ & - \\
\hline 10 & 62 & $\mathrm{~F}$ & & 223 & - & 4.6 & - & $\mathrm{n}$ & - \\
\hline 11 & 22 & M & AVNRT & - & 206 & - & 1.6 & - & $\mathrm{p}$ \\
\hline 12 & 43 & M & Atrial fibrillation & 228 & - & 3.6 & - & $\mathrm{n}$ & - \\
\hline 13 & 34 & $M$ & & 213 & - & 3.4 & - & $\mathrm{e}$ & - \\
\hline 14 & 39 & M & & 217 & - & 3.5 & - & $\mathrm{n}$ & - \\
\hline 15 & 53 & M & Atrial fibrillation & - & 205 & - & 0.9 & - & $\mathrm{p}$ \\
\hline 16 & 45 & $\mathrm{~F}$ & AVNRT & - & 212 & - & 2.2 & - & $\mathrm{p}$ \\
\hline 17 & 61 & M & & 208 & 211 & 6.0 & 0.9 & $\mathrm{e}$ & $\mathrm{e}$ \\
\hline 18 & 46 & $\mathrm{~F}$ & AVRT, atrial tachycardia & 245 & 223 & 3.9 & 2.1 & $\mathrm{n}$ & $\mathrm{p}$ \\
\hline 19 & 68 & M & Coronary artery disease & 221 & - & 6.4 & - & $\mathrm{n}$ & - \\
\hline 20 & 48 & M & Sick sinus syndrome & - & 237 & - & 1.0 & - & $\mathrm{p}$ \\
\hline 21 & 69 & M & $\mathrm{HCM}$ & 198 & - & 3.8 & - & $\mathrm{e}$ & - \\
\hline 22 & 67 & M & & 221 & 221 & 5.4 & 1.3 & $\mathrm{n}$ & $\mathrm{p}$ \\
\hline 23 & 62 & $M$ & & 241 & - & 4.5 & - & $\mathrm{n}$ & - \\
\hline 24 & 46 & M & & 218 & 206 & 8.0 & 1.4 & $\mathrm{n}$ & $\mathrm{p}$ \\
\hline 25 & 78 & $\mathrm{M}$ & & 207 & - & 11.7 & - & $\mathrm{e}$ & - \\
\hline 26 & 45 & M & Atrial septal defect & 202 & - & 5.5 & - & $\mathrm{n}$ & - \\
\hline 27 & 45 & M & & 180 & - & 4.2 & - & $\mathrm{n}$ & - \\
\hline 28 & 60 & $\mathrm{~F}$ & & 203 & - & 5.4 & - & $\mathrm{n}$ & - \\
\hline 29 & 58 & $\mathrm{~F}$ & & - & 214 & - & 1.8 & - & $\mathrm{e}$ \\
\hline 30 & 74 & $\mathrm{M}$ & & 236 & - & 5.3 & - & $\mathrm{n}$ & - \\
\hline 31 & 64 & M & Atrial tachycardia & 213 & 219 & 9.0 & 1.3 & $\mathrm{n}$ & $\mathrm{p}$ \\
\hline 32 & 74 & $\mathrm{~F}$ & Atrial fibrillation & - & 217 & - & 1.8 & - & $\mathrm{e}$ \\
\hline 33 & 20 & M & & 186 & 184 & 7.5 & 1.7 & $\mathrm{n}$ & $\mathrm{e}$ \\
\hline 34 & 57 & M & & 197 & - & 3.0 & - & $\mathrm{e}$ & - \\
\hline 35 & 83 & $\mathrm{~F}$ & AVNRT & 219 & 225 & 4.4 & 1.8 & $\mathrm{n}$ & $\mathrm{p}$ \\
\hline 36 & 74 & M & Coronary artery disease & 204 & 248 & 4.8 & 1.7 & $\mathrm{n}$ & $\mathrm{n}$ \\
\hline 37 & 44 & $\mathrm{M}$ & Aortic regurgitation & 237 & 237 & 5.3 & 1.4 & $\mathrm{n}$ & $\mathrm{e}$ \\
\hline Mean (SD) & $56(15)$ & & & $215(22)$ & $215(16)$ & $5.7(2.5)$ & $1.5(0.4)^{\star}$ & & \\
\hline
\end{tabular}

${ }^{\star} \mathrm{p}<0.00001$ compared with aVF/lead I flutter wave amplitude ratio in counterclockwise atrial flutter.

AFL, atrial flutter; AVNRT, atrioventricular nodal reentrant tachycardia; AVRT, atrioventricular reciprocating tachycardia; CCW, counterclockwise; CL, cycle length; CW, clockwise; e, equivocal; FW, flutter wave; HCM, hypertrophic cardiomyopathy; n, negative; $\mathrm{p}$, positive.

coronary sinus. A duodecapolar "Halo" catheter (Cordis-Webster, Baldwin Park, California, USA) with $10 \mathrm{~mm}$ paired spacing was inserted for mapping of the lateral and anterior right atrial wall and the interatrial septum. The first pair of electrodes (Halo 1) was positioned at the low anterior lateral right atrium and the 10th pair (Halo 10) was positioned near the coronary sinus os (fig 1A). Quadripolar roving catheters (initially at the high right atrium and right ventricular apex) were used for pacing and entrainment study at either side of the isthmus (low anterior right atrium and coronary sinus os). Surface ECG and intracardiac electrograms were recorded and displayed by a multichannel recorder (CardioLab 3.1 system, Prucka Engineering Inc, Houston, Texas, USA). The limb leads were placed at bilateral arms and hips. Pacing was performed with a programmable stimulator (Bloom Assoc, Reading, Pennsylvania, USA). For induction of atrial flutter, incremental pacing at the high right atrium, low anterior right atrium, and coronary sinus os was performed. Isthmus dependence was confirmed by: (1) termination of atrial flutter during radiofrequency ablation at the isthmus and noninducibility of atrial flutter after creation of bi-directional conduction block of the isthmus ${ }^{8-910-11}$; and/or (2) the demonstration of entrainment with concealed fusion and with a return cycle equal to flutter cycle length and a short spike to last entrained beat interval near the coronary sinus os (for counterclockwise atrial flutter), or at the low anterior right atrium (for clockwise atrial flutter) (fig 1B and C). The ablation was applied during atrial flutter and could terminate the atrial flutter in all patients. The flutter circuit orientation in the right atrium was determined by the activation sequence recorded by the Halo catheter.

ECG-ATRIAL ELECTROGRAM CORRELATION

Atrial electrograms and 12 lead surface ECG were recorded simultaneously. A period of at least 3 to 1 atrioventricular block during atrial flutter was chosen for flutter wave analysis. Adenosine was given if persistent 2 to 1 block was noted. The 12 lead ECG was read and measured at $25 \mathrm{~mm} /$ second paper speed. The flutter wave amplitude in the 12 leads was measured from the peak to the nadir on the amplified electrograms and averaged from five flutter waves using the electronic calipers on the CardioLab system. The flutter wave polarity in the inferior leads was determined by four independent cardiologists who did not know the results of invasive electrophysiological studies. The flutter wave polarity was considered equivocal if a consensus could not be reached among the four cardiologists.

\section{STATISTICS}

Non-parametric data were analysed by the Student's $t$ test. Because there were 47 atrial 

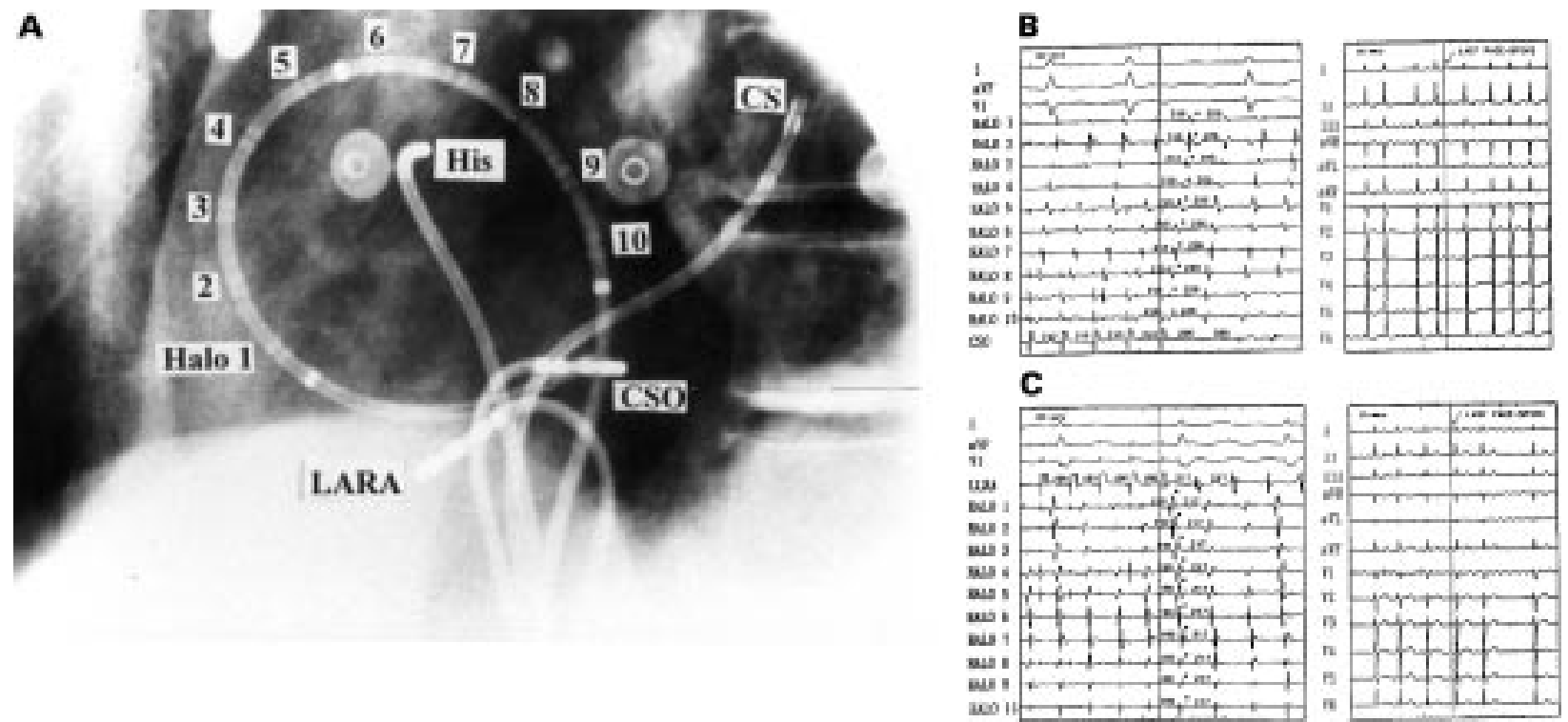

Figure 1 Fluoroscopic picture and electrograms during mapping and entrainment study. (A) Position of the Halo catheter in the right atrium (60 left anterior oblique projection). (B) Entrainment study in a patient with counterclockwise atrial flutter. The atrial activation sequence was ascending from Halo 10 to Halo 1, indicating a counterclockwise circuit in the right atrium (left). The ECG flutter waves were the same during atrial flutter and during pacing near the coronary sinus os, indicating entrainment with concealed fusion (right). The stimulus to last entrained beat interval was short and the return cycle equalled flutter cycle length, indicating entrainment at the exit site. (C) Entrainment study in a patient with clockwise atrial flutter. The atrial activation sequence was descending from Halo 1 to Halo 10, indicating a clockwise circuit (left). The ECG flutter waves were the same during atrial flutter and during pacing near the low anterior right atrium, indicating entrainment with concealed fusion (right). Entrainment with a short stimulus to last entrained beat interval and a return cycle equal to flutter cycle length indicated exit site at the low anterior right atrium. *, last entrained beat; CS, coronary sinus; CSO, coronary sinus os; His, His bundle region; LARA, low anterior right atrium.

flutter patterns in 37 patients, with 10 patients having both types of atrial flutters, each of these 10 patients had two observations. $p$ values $<0.05$ were considered significant.

\section{Results}

In the 37 patients, there were 30 counterclockwise atrial flutters and 17 clockwise atrial flutters. Ten patients had both forms of atrial flutter. All atrial flutters were isthmus dependent and were eliminated by creating a line of conduction block at the isthmus. Clinically, 33 patients had only one atrial flutter pattern and four patients had two atrial flutter patterns. All the clinical atrial flutter patterns $(4 \times 2+33=41)$ could be reproduced with similar flutter wave morphology during the electrophysiological study. In addition, six clockwise atrial flutter patterns were induced at the electrophysiological study in six patients who had counterclockwise atrial flutter clinically.

ELECTROPHYSIOLOGICAL STUDY

The intracardiac electrograms recorded by the Halo catheter showed two distinct patterns. In counterclockwise atrial flutter, the electrograms showed a completely ascending wavefront from Halo 10 to Halo 1, whereas clockwise atrial flutter was characterised by a completely descending wavefront from Halo 1 to Halo 10. In counterclockwise atrial flutter, the area near the coronary sinus os was the exit site of the slow conduction zone as revealed by the entrainment study (fig 1B). There was caudocranial activation in the interatrial septum followed by craniocaudal activation in the right atrial free wall. In clockwise atrial flutter, the low anterior right atrium was the exit site and the electrical activity had caudocranial trans-
Table 2 ECG flutter wave amplitude (in $\mathrm{mV}$ ) in both types of atrial flutter

\begin{tabular}{llll}
\hline ECG lead & CCW & $C W$ & p value \\
\hline I & $0.06(0.02)$ & $0.09(0.03)$ & 0.00035 \\
II & $0.28(0.07)$ & $0.16(0.05)$ & 0.00001 \\
III & $0.25(0.07)$ & $0.13(0.05)$ & 0.00001 \\
aVR & $0.15(0.05)$ & $0.12(0.03)$ & 0.027 \\
aVL & $0.12(0.04)$ & $0.09(0.04)$ & 0.016 \\
aVF & $0.27(0.06)$ & $0.13(0.03)$ & 0.00001 \\
V1 & $0.14(0.05)$ & $0.13(0.05)$ & NS \\
V2 & $0.09(0.04)$ & $0.09(0.07)$ & NS \\
V3 & $0.09(0.04)$ & $0.07(0.06)$ & NS \\
V4 & $0.09(0.05)$ & $0.07(0.05)$ & NS \\
V5 & $0.09(0.05)$ & $0.08(0.04)$ & NS \\
V6 & $0.09(0.04)$ & $0.08(0.03)$ & NS \\
\hline
\end{tabular}

Values are mean (SD)

CCW, counterclockwise; CW, clockwise.

mission in the right atrial free wall followed by craniocaudal transmission in the interatrial septum (fig 1C). In general, rapid pacing at the coronary sinus os induced counterclockwise atrial flutter, whereas rapid pacing at the low anterior right atrium induced clockwise atrial flutter. These observations are similar to those reported by Olgin and co-workers. ${ }^{12}$ Rapid pacing at the high right atrium could induce both types of atrial flutter. The flutter circuit direction was not related to the site of rapid pacing if transient atrial fibrillation intervened between rapid pacing and atrial flutter.

ECG COMPARISON BETWEEN COUNTERCLOCKWISE AND CLOCKWISE ATRIAL FLUTTERS

Counterclockwise and clockwise atrial flutters did not differ significantly in the flutter wave cycle length (table 1). The flutter wave polarity in the inferior leads appeared negative in 24, positive in one, and equivocal in five of the counterclockwise atrial flutters whereas the polarity appeared negative in one, positive in 10 , and equivocal in six of the clockwise atrial 

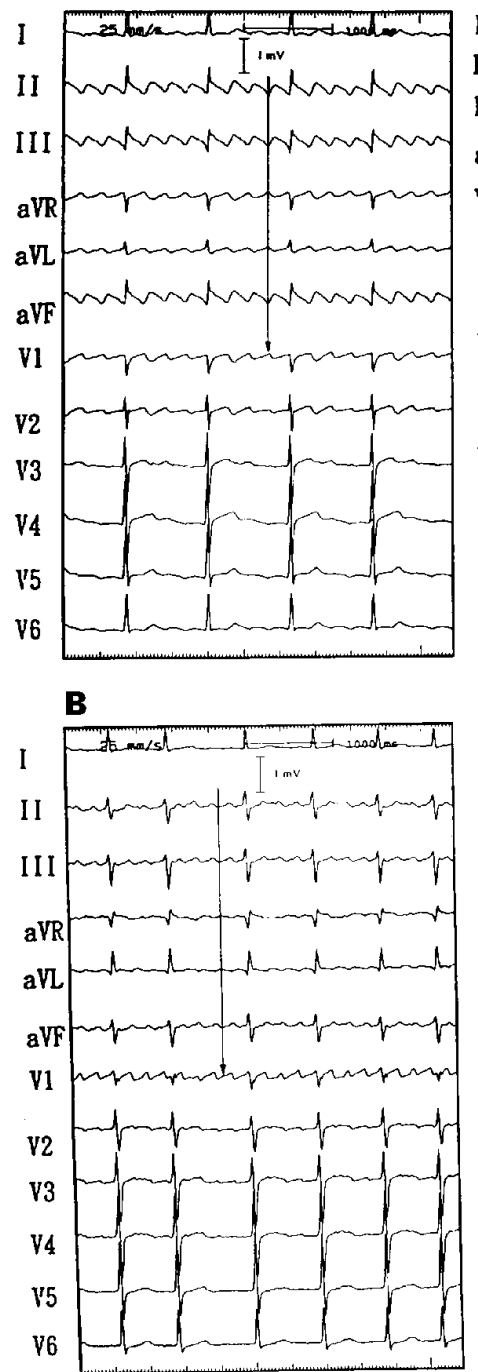

I

II

$$
\begin{aligned}
& \mathrm{aVF} \\
& \mathrm{V} 1
\end{aligned}
$$

HALO

HALO 2

HALO 3

HALO 4

HALO 5

HALO 6

HALO 7

HALO 8

HALO

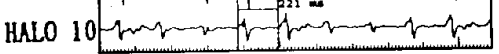

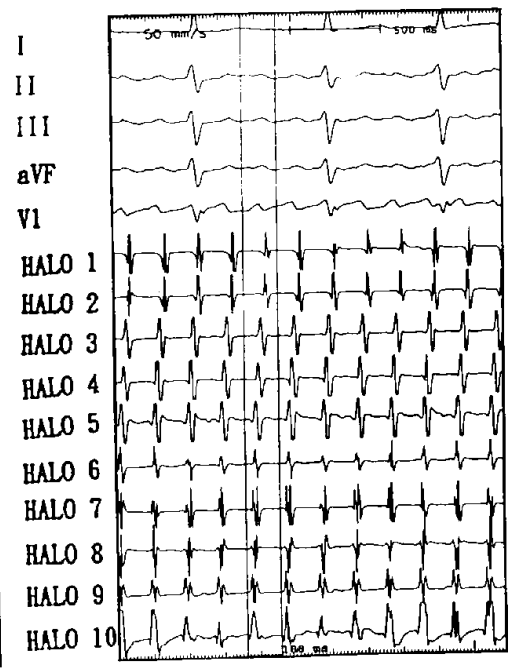

Figure 2 Twelve lead ECG (left panel; $25 \mathrm{~mm} /$ second) and intracardiac electrograms (right panel; $50 \mathrm{~mm} / \mathrm{second}$ ) from (A) patient 19 and (B) patient 5 in table 1. Both had counterclockwise atrial flutter as shown by the Halo catheter study, but patient 19 had negative flutter waves in the inferior leads and patient 5 had positive ones. However, they both had large flutter waves in the inferior leads and flat flutter waves in lead I. The aVF/lead I flutter wave amplitude ratio was $>4$ by visual inspection in both patients. The arrows indicate that the nadirs in the inferior leads corresponded to the upstrokes in V1 in both patients.

flutters. When comparing the 12 lead ECG flutter wave amplitude, we found that the flutter wave amplitude in the precordial leads was similar in both types of atrial flutter, whereas the amplitude in all limb leads differed significantly (table 2). The greatest differences were seen in the inferior leads and lead I. Counterclockwise atrial flutters had larger flutter waves in the inferior leads and smaller flutter waves in lead I than clockwise atrial flutters, and the differences were highly significant. The flutter wave amplitude in aVF was greater than $0.2 \mathrm{mV}$ in $97 \%$ (29 of 30 ) of the counterclockwise atrial flutter patterns and less than $0.2 \mathrm{mV}$ in all clockwise atrial flutter patterns. Because counterclockwise atrial flutter had large flutter waves in aVF and small ones in lead I, the aVF/ lead I flutter wave ratio had even higher differentiation power (nearly 100\%). Counterclockwise atrial flutter had a significantly higher $\mathrm{aVF} / \mathrm{lead}$ I flutter wave amplitude ratio than

clockwise atrial flutter (mean (SD) 5.7 (2.5) $v$ $1.5(0.4), \mathrm{p}<0.00001)$. All counterclockwise flutters had a ratio $>2.5$ whereas all clockwise atrial flutters had a ratio $<2.5$. When examining the 12 leads simultaneously, we found that the nadirs in the inferior leads corresponded to the upstrokes in V1 in all 30 counterclockwise atrial flutters, but corresponded to the downstrokes in V1 in all 17 clockwise atrial flutters (figs 2 and 3). Therefore, the aVF/lead I flutter wave amplitude ratio and the corresponding $\mathrm{V} 1$ points of inferior lead nadirs could be used to differentiate counterclockwise and clockwise atrial flutters with $100 \%$ accuracy in 47 atrial flutters in our study. We applied these criteria to clinical atrial flutter ECGs and both criteria could differentiate counterclockwise and clockwise atrial flutters with $100 \%$ accuracy.

\section{Discussion}

We characterised the ECG flutter waves in 47 atrial flutter patterns. All atrial flutters were isthmus dependent with either a counterclockwise or clockwise circuit in the right atrium as proved by entrainment study and by the effectiveness of isthmus ablation. Our study demonstrated that atrial flutters with negative or positive flutter waves in the inferior leads did not correspond to counterclockwise and clockwise atrial flutter, respectively. To differentiate these two forms of atrial flutter, we propose new ECG criteria with high accuracy for the differentiation between counterclockwise and clockwise atrial flutters.

FLUTTER WAVE POLARITY IN THE INFERIOR LEADS Classically, atrial flutter with negative flutter waves in the inferior leads is called typical, common, or type I atrial flutter and is believed to be isthmus dependent with a counterclockwise rotating circuit in the right atrium. Atypical, uncommon, or type II atrial flutter has the same circuit in the opposite direction. Therefore, the flutter wave polarity in the inferior leads is positive and opposite to that in typical (counterclockwise) atrial flutter. However, our results demonstrated that differentiation by this classic concept is not reliable. First, the flutter waves in the inferior leads might appear positive in counterclockwise atrial flutter, and atrial flutter with negative flutter waves in the inferior leads could have a clockwise rotating circuit. Similar findings have been reported previously. Cosio et al reported their mapping results in seven patients with the "rare" form of atrial flutter (positive flutter waves in the inferior leads). They found that two of the seven patients had a counterclockwise rotating circuit in the right atrium. ${ }^{13}$ Furthermore, we found that the interpretation of surface ECG flutter wave polarity was sometimes difficult and visual judgment was subjective. In our study, a consensus could not be reached among the four cardiologists in 11 of the 47 atrial flutters. The undulating pattern can be interpreted either as a "W" shape with negative flutter waves or as an " $M$ " shape with positive flutter waves because there is no definite starting point or isoelectric line. Therefore, the flutter wave 
A

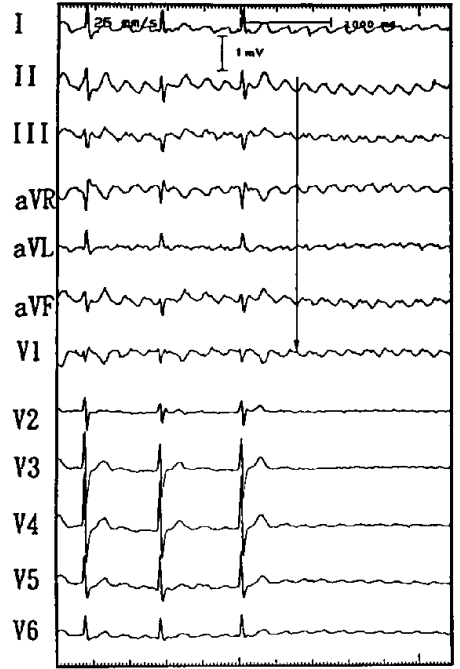

B
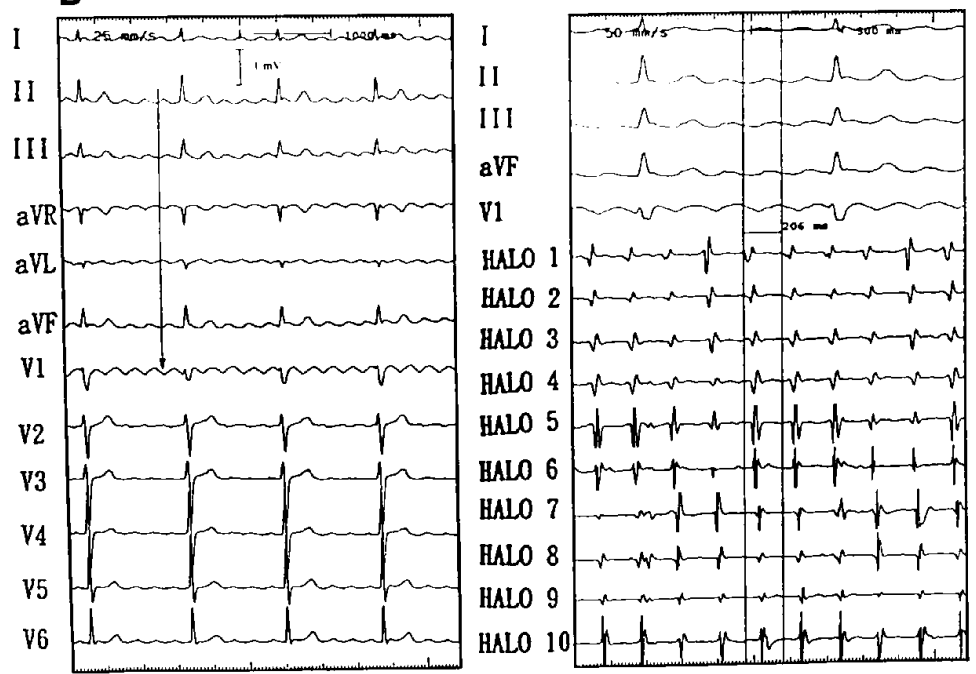

Figure 3 Twelve lead ECG (left panel; $25 \mathrm{~mm} /$ second) and intracardiac electrograms (right panel; $50 \mathrm{~mm} / \mathrm{second}$ ) from (A) patient 17 and (B) patient 24 in table 1. Both had clockwise atrial flutter as shown by the Halo catheter study, but patient 17 had equivocal flutter wave polarity in the inferior leads (interpreted as positive by two cardiologists and negative by two other cardiologists) and patient 24 had positive flutter waves in the inferior leads. However, they both differed from counterclockwise atrial flutter by virtue of a smaller aVF/lead I flutter wave amplitude ratio (<2.5). The arrows indicate that the nadirs in the inferior leads corresponded to the downstrokes in V1 in both patients.

polarity in the inferior leads is not a good predictor of the flutter circuit direction.

DIFFERENTIATION BETWEEN COUNTERCLOCKWISE AND CLOCKWISE ATRIAL FLUTTERS

We propose the following criteria for the differentiation between counterclockwise and clockwise atrial flutters. First, the aVF/lead I flutter wave amplitude ratio is $>2.5$ in counterclockwise atrial flutter but $<2.5$ in clockwise atrial flutter. Second, the nadirs in the inferior leads correspond to the upstrokes in V1 in counterclockwise atrial flutter but correspond to the downstrokes in V1 in clockwise atrial flutter. An amplitude in aVF $>0.2 \mathrm{mV}$ was also highly suggestive of counterclockwise atrial flutter, with a sensitivity of $97 \%$ (29 of 30). ECG amplitude is influenced by many non-cardiac factors such as lung volume, body mass, etc. These factors can be cancelled by calculating the amplitude ratio and we found that the $\mathrm{aVF} /$ lead I ratio had an even higher differentiation power. The flutter wave amplitude was measured using the electronic calipers in the CardioLab system in the study. There may be concern about measuring the amplitude on standard 12 lead ECG. We found that the flutter wave amplitude in lead I is very small in some patients and it is difficult to make an accurate measurement from a standard 12 lead ECG. However, we also found that all such ECG patterns were counterclockwise atrial flutters and the aVF/lead I ratio was far greater than 2.5 by visual inspection (usually $>4$; fig 2). For ECG patterns with an aVF/lead I ratio near to 2.5, the flutter wave in lead I was larger and measurable. Therefore, clinicians can correctly classify the flutter by applying our criteria to a standard 12 lead ECG.

We propose a speculative explanation for the ECG differences between counterclockwise and clockwise atrial flutters. In counterclockwise atrial flutter, the exit site of the slow conduction zone is near the coronary sinus os, which is located at the lowermost part of the interatrial septum. The bulk of right and left atria are both activated in an inferior to superior direction. The $\mathrm{P}$ waves are large in the inferior leads because the direction of activation is parallel to these leads and the $P$ waves are flat in lead I because the activation direction is perpendicular to lead I. In clockwise atrial flutter, atrial activation originates from the low anterior lateral corner of the right atrium. The right atrium is activated before the left atrium and the atrial mass is activated in a right to left direction. Therefore, clockwise atrial flutter has larger flutter waves in lead I and smaller flutter waves in the inferior leads than does counterclockwise atrial flutter. At the nadir in the inferior leads, the electrical activity is at the high right atrium in both types of atrial flutters. At this point, the electrical activity spreads from the high right atrium towards the low anterior right atrium in counterclockwise atrial flutter and, therefore, corresponds to the upstroke in V1. In clockwise atrial flutter, the electrical activity spreads from the high right atrium to the coronary sinus os and, therefore, corresponds to the downstroke in V1. The results of our intracardiac electrogram study contribute to the understanding of the ECG differences between counterclockwise and clockwise atrial flutters, and also explain why the above criteria might be used to differentiate reliably between counterclockwise and clockwise atrial flutters.

The current classification of atrial flutter is diverse and sometimes confusing. There have been various classification methods based on flutter wave polarity, atrial rate, response to overdrive, and other criteria. ${ }^{514}{ }^{15}$ There are also many terms for the description of atrial flutter, including typical and atypical, common and uncommon, type I and type II, and classic and rare. None of these classifications can predict the flutter mechanism reliably, and the interobserver consistency with regard to flutter wave polarity is not good. We recommend the following classification. Atrial flutters can be divided into isthmus dependent and isthmus independent, 
and the former can be divided into counterclockwise and clockwise atrial flutters. Because isthmus independent atrial flutter is extremely rare (according to the literature and our results), our differentiation criteria can be applied to most atrial flutters. Counterclockwise and clockwise atrial flutters can be differentiated by simple visual examination of a routine 12 lead ECG tracing. This classification is not only simple and objective but also correlates reliably with the underlying flutter mechanism.

COMPARISON WITH PREVIOUS STUDIES REGARDING ECG PATTERN IN CLOCKWISE ATRIAL FLUTTERS

The ECG characteristics have been addressed in several previous reports. ${ }^{16-18}$ Saoudi et al reported a classic "sawtooth pattern" in 14 of 18 clockwise atrial flutters. ${ }^{16}$ They also reported that the flutter wave amplitude in the inferior leads was smaller in clockwise than in counterclockwise atrial flutters; our study provides a cut off value for differentiation. Tai et al reported that in 30 clockwise atrial flutters the flutter wave polarity in the inferior leads was positive in 10 and biphasic in the other $20 .{ }^{17}$ This agrees with our conclusion that flutter wave polarity is not a good indicator of underlying flutter wave rotating direction. Kalman et al proposed that a notched flutter wave in the inferior leads was a stereotypical feature of clockwise atrial flutter. ${ }^{18}$ However, this was seen in only half of the clockwise atrial flutters in Saoudi's report. ${ }^{16}$ A notched flutter wave in clockwise atrial flutters was not a constant finding in our study group either (fig 3B), and a notched flutter wave was also seen in aVF in counterclockwise atrial flutter (fig 2B). Flutter wave polarity in V1 was addressed in two reports. ${ }^{16}{ }^{18}$ There is a tendency for positive waves in V1 in counterclockwise atrial flutter and negative waves in clockwise atrial flutter. However, a biphasic or continuously undulating pattern is sometimes seen. We discourage the use of positive or negative flutter waves for classification because their identification is subjective and difficult, especially for flutter waves with an undulating pattern.

\section{LIMITATIONS}

Isthmus independent atrial flutters were not observed in our study population of 37 consecutive patients with 47 atrial flutters. Most (31 of 37) of the patients had no organic heart disease, which could account for this absence of isthmus independent atrial flutters. The clinical usefulness of differentiating counterclockwise and clockwise atrial flutters might be somewhat limited with regard to electrophysiological study and catheter ablation because the electrophysiological approach, catheter ablation method, and end point are similar for counterclockwise and clockwise atrial flutters. However, we think these criteria can remove the ambiguity of classic terms such as typical versus atypical and common versus uncommon atrial flutter. Isthmus independent atrial flutter is very rare as reported in the literature, and comprises a heterogeneous group in which the mechanism and ECG characteristics remain unclear. ${ }^{1420}$ This topic deserves future investigation.

Mapping of the left atrium was not complete and was limited. The coronary sinus mapping catheter recorded electrical activity only from the part of the left atrium near the atrioventricular groove. However, despite the limited left atrial mapping sites, we were still able to identify the flutter mechanism confined in the right atrium and to perform the catheter ablation successfully.

Presented in part at the 18th Annual Scientific Session of the North American Society of Pacing and Electrophysiology in New Orleans, Louisiana in May 1997.

1 Jolly WA, Ritchie WT. Auricular flutter and fibrillation. Heart 1911;2:177-221.

2 Klein GJ, Guiraudon GM, Sharma AD, et al. Demonstration of macro-reentry and feasibility of operative therapy in
the common type of atrial flutter. Am $\mathcal{F}$ Cardiol 1986;57: 587-91.

3 Beckman K, Lin HT, Krafchek J, et al. Classic and concealed entrainment of typical and atypical atrial flutter. PACE 1986;9:826-35.

4 Olshansky B, Okumura K, Hess PG, et al. Demonstration of an area of slow conduction in human atrial flutter. $\mathcal{F} \mathrm{Am}$ Coll Cardiol 1990;16:1639-48.

5 Cosio FG, Lopez GM, Goicolea A, et al. Electrophysiologic Cosio FG, Lopez GM, Goicolea A, et al. Electrophysiolo
studies in atrial flutter. Clin Cardiol 1992;15:667-73.

6 Olshansky B, Wilber DJ, Hariman RJ. Atrial flutter: update on the mechanism and treatment. PACE 1992;15:2308-35. Waldo AL. Atrial flutter: new directions in management and mechanism. Circulation 1990;81:1142-3.

8 Cosio FG, Lopez-Gil M, Goicolea A, et al. Radiofrequency ablation of the inferior vena cava-tricuspid valve isthmus in common atrial flutter. Am f Cardiol 1993;71:705-9.

9 Feld GK, Fleck P, Chen PS, et al. Radiofrequency catheter ablation for the treatment of human type 1 atrial flutter. Circulation 1992;86:1233-40.

10 Chen SA, Chiang CE, Wu TJ, et al. Radiofrequency catheter ablation of common atrial flutter: comparison of electrophysiologically guided focal ablation technique and linear ablation technique. $\mathcal{F}$ Am Coll Cardiol 1996;27:860-8.

11 Kirkorian G, Moncada E, Chevakier P, et al. Radiofrequency catheter ablation of atrial flutter. Efficacy of an anency catheter ablation of atrial flutter. Efficacy of an 14.

12 Olgin JE, Kalman JM, Saxon LA, et al. Mechanism of initiation of atrial flutter in humans: site of unidirectional block nd direction of rotation. 7 Am Coll Cardiol 1997;29:36784

13 Cosio FG, Goicolea A, Lopez-Gil M, et al. Atrial endocardial mapping in the rare form of atrial flutter. Am $\mathcal{F}$ Cardiol 1990;66:715-20.

4 Wells JL, MacLean WAH, Lames TN, et al. Characterization of atrial flutter. Studies in man after open heart surgery using fixed atrial electrodes. Circulation 1979;60:665-3.

15 Lesh MD, Kalman JM. To fumble flutter or tackle "tach"? Toward updated classifiers for atrial tachyarrhythmias. $f$ Cardiovasc Electrophysiol 1996;7:460-6.

16 Saoudi N, Nair M, Abdelazziz A, et al. Electrocardiographic patterns and results of radiofrequency catheter ablation of clockwise type I atrial flutter. $\mathcal{F}$ Cardiovasc Electrophysiol 1996;7:931-42.

17 Tai CT, Chen SA, Chiang CE, et al. Electrophysiologic characteristics and radiofrequency catheter ablation in patients with clockwise atrial flutter. $\mathcal{f}$ Cardiovasc Electropatients with clockwise

18 Kalman JM, Olgin JE, Saxon LA, et al. Electrocardiographic and electrophysiologic characterization of atypical atrial flutter in man: use of activation and entrainment mapping and implications for catheter ablation. $\mathcal{F}$ Cardiovasc Electropysiol 1997;8:121-44.

19 Poty H, Nair M, Anselme F, et al. Electrophysiologic features and detailed endocardial mapping of left atrial flutter [abstract]. Circulation 1996;94(suppl):I-555.

20 Kall J, Rubenstein D, Kopp D, et al. Characterization and catheter ablation of right atrial free wall atypical atrial flutter [abstract]. Circulation 1996;94(suppl):I-676. 